\title{
Design of a microfluidic paper-based device for analysis of biomarkers from urine samples on diapers
}

\author{
Adriana Couto and Tao Dong, Member, IEEE
}

\begin{abstract}
Among all infections, urinary tract infections (UTI) are one of the most common. Nowadays the procedures to analyze urine and consequently detect UTI are often painful and time-consuming. Recent studies about microfluidic paper-based devices have developed the interest of researchers due their outstanding characteristics. In this paper is presented a novel design for a microfluidic paper-based device for screening and analysis of multiple biomarkers from urine samples on diapers. The device consists on a set of eight layers. It was designed based on the previous attempts to improve and overcome some problems detected as the continuous entrance of fluids, the possibility of contamination and the invalidity of results due to communication between different reagent pads. One approach was create a "self-locking" mechanism that closes the sample inlet in approximately four minutes solving the first two problems. Furthermore, is important that comfort is guaranteed, hence a device with a total thickness of $5,3 \mathrm{~mm}$ is presented. This device can keep the results for eight hours and can be used as a low-cost and more effective alternative than conventional methods being a strategy with potential for the diagnostic and analysis of biological samples in the future improving healthcare.
\end{abstract}

\section{INTRODUCTION}

Urinary tract infections (UTI) are very common and affect males and females. Due to anatomy and physiology, women are the most affected by these bacterial infections and it is estimated that almost one out of three of them will experience one or more episodes of UTI by the age of 24 . However, the population group with the highest prevalence of UTI are the elderly [1]-[4].

Conventional methods used for detection of UTI are urinalysis using dipsticks, microscopic examination and urine culture [4]. Dipsticks are accurate and a low-cost method however is required a great amount of sample which can be extremely hard to get [1], [5]. Microscopic examination is a

Adriana Couto is with Institute of Applied Micro-Nano Science and Technology - IAMNST, Chongqing Key Laboratory of Colleges and Universities on Micro-Nano Systems Technology and Smart Transducing, Chongqing Engineering Laboratory for Detection, Control and Integrated System, National Research Base of Intelligent Manufacturing Service, Chongqing Technology and Business University, Nan'an District, Chongqing 400067, China; with Department of Biological Engineering, University of Minho, Campus de Gualtar, 4710-057 Braga, Portugal; and with Department of Microsystems - IMS, Faculty of Technology, Natural Sciences and Maritime Sciences, University College of Southeast Norway, Postboks 235, $3603 \quad$ Kongsberg, Norway. (e-mail: Adriana_mssc@hotmail.com).

Tao Dong is with Department of Microsystems - IMS, Faculty of Technology, Natural Sciences and Maritime Sciences, University College of Southeast Norway, Postboks 235, 3603 Kongsberg, Norway. (corresponding author to provide phone: +47 33037731; email: tao.dong@hbv.no). complement to dipstick urinalysis and is requested when an abnormal result on dipstick is obtained. There are some associated-costs both personal and equipment that is why it is crucial to reduce the requests or find an alternative more cost-effective for this option [6]. Urine culture provides quantitative result so is the gold standard for the diagnosis of UTI. However, there are some substantial costs associated and it takes between 24 and 48 hours to get the results. Moreover, is required a large volume of sample and contamination may occur. When the protocol for sample collection is not strictly followed lead to a waste of time, money and resources [7], [8].

Urinalysis is very used in clinical practice for detection of UTI and should be a practical, fast, cheap, sensitive and specific test leading to the right treatment for each case [1]. The parameters analyzed during this kind of test can be $\mathrm{pH}$, specific gravity, color, blood, leukocyte esterase, glucose, ketone, protein and nitrite [9]. Besides UTI, urinalysis can be useful to detect other diseases like chronic renal diseases, cardiovascular diseases and liver problems [10].

Diapers are often used in patients who suffer from urinary incontinence and may be used for sample collection that is often a painful and invasive procedure, such as the transurethral catheterization and the supra-pubic bladder aspiration, or even impossible in this group of patients [11], [12].

The search for a simple and low-cost approach to disease diagnostics can make healthcare available globally. Microfluidic paper-based devices are an emerging subject due to their capacity for preliminary diagnostics of diseases and analysis of biological samples. They are normally inexpensive, easy-to-operate, portable and require small sample volumes. Paper substrates present two great advantages: (1) compatibility with biological samples; (2) wicking capability. Paper-based microfluidic devices are based on patterns of microchannels forming hydrophilic and hydrophobic regions using, for instance, inkjet etching, plasma etching, photolithography or wax printing. The last is considered an inexpensive and rapid procedure encompassing only two simple steps: first patterning the microchannels with wax then melt the wax creating hydrophobic barriers. The melting step of wax printing limits the resolution, but is a good option when the dimensions are sufficiently large [13], [14].

Regarding colorimetric assays, they are simple to execute, allows an analysis by both naked eye or using a smartphone application and it is possible to obtain quantitative results [15], [16].

In this paper, a novel design for a microfluidic paper-based device for screening and analysis of multiple biomarkers from urine samples on diapers is presented. 


\section{DESIGN OF DEVICE}

\section{A. General description}

A device that can be attached on a diaper to collect and transport a urine sample to colorimetric reaction pads through capillary action in absorbent media was designed. It provides a quantitative result and is capable to retain the biological fluid in a non-dry state for eight hours due to the autonomous "self-locking" and the plastic cover on it that isolate the test area from the external environment. When the diaper is removed from the patient, the result can be read through the transparent plastic cover, however, for a more accurate result the colorimetric analysis can be conducted by a smartphone application. In Fig. 1 is shown, in reverse way, a set of eight layers that compose the device with a total thickness of $5,3 \mathrm{~mm}$. Except the surface layer that is composed by three sub-layers and the test layer that is composed by three different parts: filter paper, reagent pads and wax, all the other layers are a single piece.

\section{B. Challenges and solutions}

During the conception of device some challenges were faced. The first one regarding the isolation of test conditions from the external environment: it was necessary to find a way to stop the entrance of any liquid when there is enough amount of urine to proceed with the test avoiding contamination. To overcome this issue a "self-locking" mechanism was proposed.

Results on reagent pads are based on a colorimetric reaction between urine biomarker and reagents in pads. The problem experienced is the same as on dipsticks: communication between pads can invalidate the results. To avoid the interference between pads, hydrophobic channels on filter paper and a layer with six holes, named frames from now on, with the pads dimensions were created.

\section{Sample flow and device composition}

The sample flow is represented in Fig. 2. Urine is collected in surface layer (see 1, Fig. 1) and passes through the inlet present in reference layer (see 2, Fig. 1). Then it passes through two more round holes; the first one is on the structure layer (see 3, Fig. 1) and the second one on the barrier layer (see 4, Fig. 1) until it reaches the filter paper in test layer (see 6, Fig. 1). In this layer, the urine flow occurs

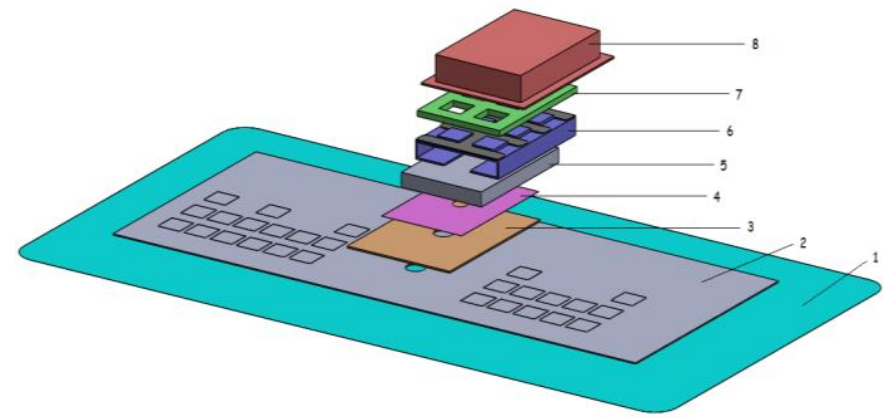

Figure 1. Set of eight layers that compose the device. (1) Surface layer; (2) Reference layer; (3) Structure layer; (4) Barrier layer; Impermeable layers: (5) Hydrogel host layer and (7) Frame layer; (6) Test layer; (8) Plastic cover. by capillary action to reagent pads. The filter paper is wrapped around an impermeable layer (see 5, Fig. 1) with a round hole to place a hydrogel constituting a "self-locking" mechanism together with the barrier layer. Another impermeable layer (see 7, Fig. 1) is present on top of test layer and fits on the pads due to the frames on it. The plastic cover (see 8, Fig. 1) protects all layers from the environment around and allows the visualization of reagent pads.

\section{Materials and function of each layer}

The surface layer was made by three sub-layers: two of them (sub-layer 1 and 2) made of non-woven material and the third made of Kraft, making a total thickness of $0,3 \mathrm{~mm}$. They are bonded together using heat bonding on two edges (the ones with biggest length). The sub-layer 2, covered by thin medical pressure-sensitive adhesive film, such as acrylate, is glued with sub-layer 3. Sub-layer 3 has a rectangular hole on center where the reference layer is placed. The surface layer is permeable to biological fluid and can quickly absorb the urine. Moreover, it acts as a barrier to prevent liquid from penetrating backwards. This layer is in contact with patient's skin and its absorbent capacity, minimize the possibility of bacterial growth and consequently avoid the emergence of UTI.

The reference layer has a thickness of $0,2 \mathrm{~mm}$ and a round hole that collects fluid and guides it to structure layer. The reference layer was made by printing high quality reference colors on paper and then applying a coat of transparent hydrophobic material, for example, polyvinyl chloride (PVC), or other transparent impermeable plastic to protect the colors from the environment. The reference layer's colors allow, for comparison, a direct read-out of results. However, a smartphone application can be used for objective analysis.

The structure layer is impermeable to biological fluid and made of $0,3 \mathrm{~mm}$ Poly (methyl methacrylate) (PMMA). It comprises an inlet located at the center, which collect the fluid from reference layer and transport it to barrier layer. The structure layer gives stability to the device and avoids the contact of the wet region with the reference layer.

The barrier layer was made with a flexible and non-permeable plastic, such as, cellophane (thickness of $0,1 \mathrm{~mm}$ ) with a hole on center. The function of this layer is to assist the "self-locking" mechanism, i.e., to stop the fluid entrance and avoid contamination after urine absorption.

The test layer was made of an absorbent porous medium, which may be filter paper. The test layer comprises a biological fluid barrier made with wax that works as a path network forming channels for liquid flow and separates the six reagent pads located within it to ensure the reliability of results. The reagent pads were glued on paper and contain chemicals that react with biomarkers from urine. The five chosen biomarkers are glucose, protein, occult blood, nitrite

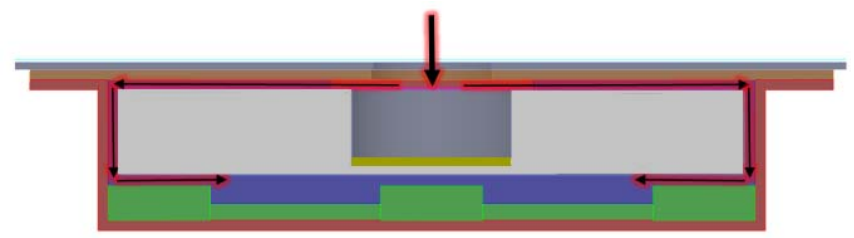

Figure 2. Sample flow in a cross-section design of on-diaper device. 
and leukocyte esterase. The other one is for the validation of wettability determining whether the biological fluid reaches all reagent pads or not. The reactions lead to a change in the color of indicators that will be compared with the reference layer's colors and permit a preliminary diagnose.

The hydrogel host layer was made of a hydrophobic material, for instance, polytetrafluoroethylene (PTFE) and prevents the collapse of test layer due to the excessive wetting. Moreover, it has a round hole to place the hydrogel. The hydrogel is employed as a swelling-based "self-locking" mechanism.

The frame layer was made of an impermeable plastic, for example, PTFE with $1 \mathrm{~mm}$ of thickness and forms an integral frame that fits on the reagent pads preventing inter-pad communication, i.e., prevent out-of-plane interference due the frames on it.

The plastic cover was made of transparent $0,3 \mathrm{~mm}$ PMMA being impermeable to biological fluid. It holds everything in place and makes possible to read results by naked eye or, in case of use a smartphone application, allows to take a picture to proceed with analysis.

\section{OPERATION MODE OF "SELF-LOCKING" MECHANISM}

As presented in Fig. 3, "self-locking" mechanism is achieved by a hydrogel together with the barrier layer. When the urine enters hydrogel absorbs it and expand, what leads to a greatly increase on hydrogel volume and drives to the inlet closing. The barrier layer made of an impermeable type of cellophane serves like a physical barrier between the filter paper and the urine sample or other fluid to prevent reabsorption by filter paper and external contamination. If one of these situations occurs, the results are not reliable. The mechanism is only functional when the hydrogel swells. The swollen hydrogel pushes the barrier layer in the reverse way of inlet flow.

(A)


Figure 3. "Self-locking" mechanism: (A) Before urine entrance; (B) Hydrogel expansion and barrier layer starts to rise; (C) Inlet closed.

\section{MATERIAL AND METHODS}

\section{A. Hydrogel specifications}

In order to find the appropriate size for hydrogel to achieve a functional "self-locking" mechanism a test was made to understand hydrogel's behavior. The swelling test was performed by swell the hydrogel in deionized water and urine at room temperature. The degree of swelling of hydrogel was measured at different times during 26 hours and with two different values of height of hydrogel $(5 \mathrm{~mm}$ and $10 \mathrm{~mm}$ ). Measurements were done in triplicate. Swelling ratio was calculated as in (1):

$$
\mathrm{D}_{\mathrm{s}}=\left[\left(\mathrm{W}_{\mathrm{h}}-\mathrm{D}_{\mathrm{h}}\right) / \mathrm{D}_{\mathrm{h}} \times 100 \%\right]
$$

where $D_{s}$ is the degree of swelling, $W_{h}$ is the weight of swollen hydrogel and $D_{h}$ is the weight of hydrogel before swelling. With this test, it was also possible to estimate the approximate time that the hydrogel takes to reach equilibrium.

\section{B. Key steps on fabrication process: test layer and hydrogel host layer together with hydrogel}

Test layer was made with a porous sheet of filter paper from Whatman (grade 1, qualitative, $\Phi 150 \mathrm{~mm}$ ) that was cut in a parallelepiped shape $(38,2 \mathrm{~mm} \times 27 \mathrm{~mm} \times 0,3 \mathrm{~mm})$. Next, bee wax was printed to create hydrophobic channels on filter paper. A simple method for printing the wax on the paper surface is screen-printing. After printing the bee wax patterns and using a hot plate or an oven, melting wax penetrates the paper and forms hydrophobic barriers (thickness of $0,2 \mathrm{~mm}$ ). The reagent pads $(5 \mathrm{~mm} \times 5 \mathrm{~mm} \times 0,5 \mathrm{~mm})$ were fabricated in Jilin Shenhua medical instruments Co., Ltd. and glued between wax barriers by chemically inert adhesives, see Fig. 4.

The hydrogel host layer was made with PTFE that was cut in parallelepiped shape (27 mm x 18,4 mm x 2,5 mm). Then a cylindrical hole was built in center of parallelepiped ( $\Phi 5 \mathrm{~mm}, 2 \mathrm{~mm}$ of height) to place the hydrogel. The extra-strong super-porous hydrogel used is a copolymer named poly (sulfopropylacrylate-co-acrylamide-co-acrylic acid-co-methylene bisacrylamide) with interpenetrating

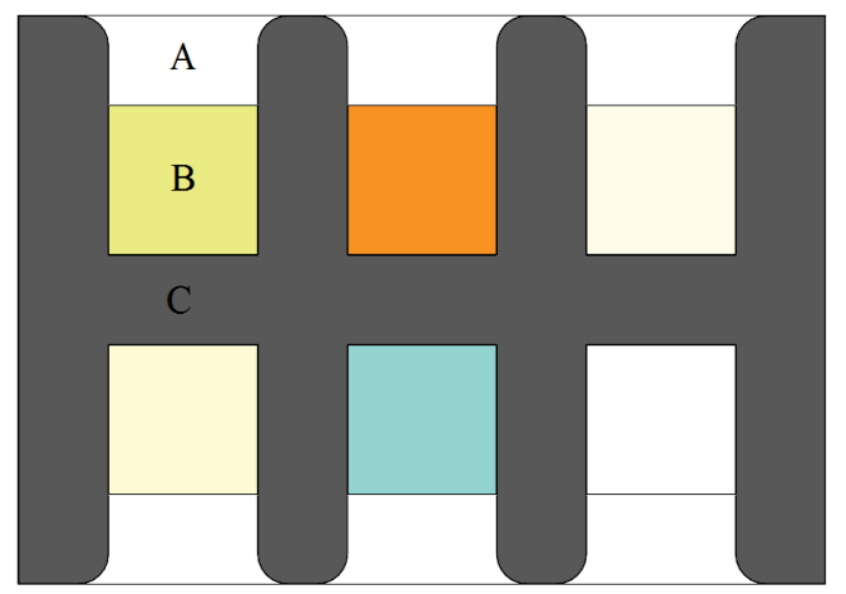

Figure 4. Top view of test layer: (A) Filter paper; (B) Reagent pad; (C) Bee wax barriers. 
network of croscarmellose sodium. Due to its extremely small dimensions ( $\Phi 5 \mathrm{~mm}, 0,2 \mathrm{~mm}$ of height), it was necessary to load it in situ in a non-porous form in the bottom of the hole, otherwise it would break or crumble.

\section{RESULTS AND DISCUSSION}

To characterize the hydrogel a swelling test was conducted. The results obtained with the calculation of swelling ratio suggest that the equilibrium is reached approximately in four minutes, i.e., the "self-locking" completes the sealing within approximately four minutes after the reagent pads being completely wetted by the urine what takes less than one minute. This is a satisfactory value because is long enough for the entry of urine sample and is not too long avoiding a second entry of liquids and contamination.

Based on hydrogel volume before and after the test, the final volume requested on the design presented and considering the direct proportionality relationship between both final volumes it was possible to calculate the height of dry hydrogel $(0,2 \mathrm{~mm})$ for a functional "self-locking" mechanism.

\section{CONCLUSION}

The promising design presented in this paper overcomes the shortcomings and some difficulties faced by the existing methods such as the contamination and the uninterrupted entrance of liquid. These problems are solved with the "self-locking" mechanism that seals the device in four minutes. This device needs only a small volume sample and is collected using a diaper what facilitates the procedure in elderly patients or disabled people since a large portion of them are incontinent and use adult diapers. It is possible to improve the efficiency of work due to the necessity of using just one device to collect, detect and analyze a urine sample and in a short period. The paper-based device distribute the urine sample into multiple spatially segregated regions allowing the test of five different biomarkers with the same sample and driven by capillary action without the need for external driving forces like pumps. It is easy-to-use, painless, portable, made with cheap materials and preserves the results for eight hours.

As future perspectives it would be very advantageous to add other useful biomarkers for diagnosis of different diseases by doing small changes on presented design.

\section{ACKNOWLEDGMENT}

The authors would like to acknowledge Dr. Nuno Miguel Matos Pires and Haakon Karlsen for technical discussions, comments and guidance during all phases of device design.

This research is mainly supported by Norsk Regionalt Forskningsfond Oslofjordfondet Prosjekt: Touchsensor for enklere og raskere urinprøvetaking og analyse (proj. no: 234972). The support from Regionale Forksningfond Oslofjordfondet forprosjekt (proj. no. 249017, 255893 and 260586) and Forprosjekt - VRIBEDRIFT Vestfold (proj. no.
252173 ) is also acknowledged. We are also thankful to the funds from National Natural Science Foundation of China (grant no. 61550110253 and 61650410655), Chongqing Research Program of Basic Research and Frontier Technology (nos. cstc2016jcyjA2161, cstc2015jcyjA20023), Science and Technology Research Program of Chongqing Education Commission (no. KJ1600604) and Chongqing Innovation Team of Colleges and Universities (no. CXTDX201601025).

\section{REFERENCES}

[1] D. Kayalp, K. Dogan, G. Ceylan, M. Senes, and D. Yucel, "Can routine automated urinalysis reduce culture requests?," Clin. Biochem., vol. 46, no. 13-14, pp. 1285-1289, 2013.

[2] R. Vasudevan, "Urinary Tract Infection: An Overview of the Infection and the Associated Risk Factors," J. Microbiol. Exp., vol. 1, no. 2, pp. 1-15, 2014.

[3] F. Montorsi, G. Gandaglia, A. Salonia, A. Briganti, and V. Mirone, "Effectiveness of a Combination of Cranberries, Lactobacillus rhamnosus, and Vitamin $\mathrm{C}$ for the Management of Recurrent Urinary Tract Infections in Women: Results of a Pilot Study," Eur. Urol., vol. 70, no. 6, pp. 912-915, 2016.

[4] Z. Arinzon, A. Peisakh, I. Shuval, S. Shabat, and Y. N. Berner, "Detection of urinary tract infection (UTI) in long-term care setting: Is the multireagent strip an adequate diagnostic tool?," Arch. Gerontol. Geriatr., vol. 48, no. 2, pp. 227-231, 2009.

[5] J. L. Young and D. E. Soper, "Urinalysis and urinary tract infection: update for clinicians.," Infect. Dis. Obstet. Gynecol., vol. 9, no. 9, pp. 249-255, 2001.

[6] M. Chen, S. Eintracht, and E. MacNamara, "Successful protocol for eliminating excessive urine microscopies: Quality improvement and cost savings with physician support," Clin. Biochem., 2016.

[7] C. W. Lam et al., "NMR-based metabolomic urinalysis: A rapid screening test for urinary tract infection," Clin. Chim. Acta, vol. 436, pp. 217-223, 2014.

[8] T. Demilie, G. Beyene, S. Melaku, and W. Tsegaye, "Diagnostic accuracy of rapid urine dipstick test to predict urinary tract infection among pregnant women in Felege Hiwot Referral Hospital, Bahir Dar, North West Ethiopia.," BMC Res. Notes, vol. 7, p. 481, Jul. 2014.

[9] J. Du et al., "Establishment and development of the personalized criteria for microscopic review following multiple automated routine urinalysis systems," Clin. Chim. Acta, vol. 444, pp. 221$228,2015$.

[10] V. Khejonnit, B. Pratumvinit, K. Reesukumal, S. Meepanya, C. Pattanavin, and P. Wongkrajang, "Optimal criteria for microscopic review of urinalysis following use of automated urine analyzer," Clin. Chim. Acta, vol. 439, pp. 1-4, 2015.

[11] M. A. M. A. Roelofs-Thijssen, M. F. Schreuder, M. Hogeveen, and A. E. van Herwaarden, "Reliable laboratory urinalysis results using a new standardised urine collection device," Clin. Biochem., vol. 46, no. 13, pp. 1252-1256, 2013.

[12] Y. Guo et al., "Heat and mass transfer of adult incontinence briefs in computational simulations and objective measurements," Int. J. Heat Mass Transf., vol. 64, pp. 133-144, 2013.

[13] Y. Lin, D. Gritsenko, S. Feng, Y. C. Teh, X. Lu, and J. Xu, "Detection of heavy metal by paper-based microfluidics," Biosensors and Bioelectronics, vol. 83. pp. 256-266, 2016.

[14] W. Dungchai, O. Chailapakul, and C. S. Henry, "A low-cost simple, and rapid fabrication method for paper-based microfluidics using wax screen-printing.," Analyst, vol. 136, no. 1, pp. 77-82, Jan. 2011.

[15] T. Yu, T.-T. Zhang, W. Zhao, J.-J. Xu, and H.-Y. Chen, "A colorimetric/fluorescent dual-mode sensor for ultra-sensitive detection of $\mathrm{Hg} 2+$, , Talanta, vol. 165, pp. 570-576, 2017.

[16] J. Li et al., "Detection of trace nickel ions with a colorimetric sensor based on indicator displacement mechanism," Sensors Actuators B Chem., vol. 241, pp. 1294-1302, 2017. 